\title{
FORCED OSCILLATIONS OF CONTINUOUS DYNAMICAL SYSTEMS
}

\author{
W. H. INGRAM
}

Introduction. The system of differential equations and boundary conditions

(a) $\frac{\partial u_{i}}{\partial x}=\Sigma a_{i j}(x) \frac{\partial u_{j}}{\partial t}+\Sigma b_{i j}(x) u_{j}, \quad \Sigma\left\{\alpha_{i j} u_{j}(a)+\beta_{i j} u_{j}(b)\right\}=0$

$(i, j=1,2, \cdots, n, a \leqq x \leqq b)$ has application in the theory of the electrical transmission line, the diffusion of heat along thin rods and around thin rings and, when some of the $u$ 's are employed to designate rates of change of other $u$ 's, to vibrating strings, bars, air columns and other dynamical systems. The system of total differential equations

$$
Y^{\prime}(x)=(\mu \mathcal{A}+\mathcal{B}) Y, \quad \mathscr{W}_{a} Y(a)+\mathscr{W}_{b} Y(b)=0,
$$

where $\mathcal{A}=\left(a_{i j}\right), \mathcal{B}=\left(b_{i j}\right), \mathscr{W}_{a}=\left(\alpha_{i j}\right), \mathscr{W}_{b}=\left(\beta_{i j}\right)$, and where $Y$ is a columnar matrix of $n$ elements each a function of $x$, may be obtained as the result of the Bernoulli-Taylor substitution $u_{i}(x, t)=\epsilon^{\mu t} y_{i}(x)$ into (a).

The system (b) has been the starting point for many researches centered around the problem of expressing an arbitrary function $f$ or, more generally, a set of functions $\left\{f_{i}\right\}$, in terms of its characteristic solutions. A solution of this problem in the simple case, having application to the uniform dissipationless vibrating string, was first obtained by Daniel Bernoulli about the year 1732 and a solution having application to the nonuniform string was first obtained by Liouville ${ }^{1}$ one hundred years later. A purportedly more rigorous treatment of Liouville's problem was given by Kneser ${ }^{2}$ in 1904. Since that date a great many papers have appeared, having to do with the system (b) under one restriction or another, the most comprehensive of which are the papers by Bliss, ${ }^{3}$ who obtained uniform convergence in his expansion theorem by requiring (b) to be "definitely" self-adjoint and by imposing a restriction on the functions $f_{i}$, and Birkhoff and Langer ${ }^{4}$ who considered the general case.

Received by the editors May 7, 1941. 269.

${ }^{1}$ Liouville, Journal de Mathématiques Pures et Appliquées, vol. 1 (1836), pp. 253,

${ }^{2}$ Kneser, Mathematische Annalen, vol. 58 (1904), p. 108.

${ }^{3}$ Bliss, Transactions of this Society, vol. 28 (1926), p. 576.

${ }^{4}$ Birkhoff and Langer, Proceedings of the American Academy of Arts and Sciences, vol. 58 (1923), p. 100. 
In their paper, Birkhoff and Langer show how the biorthogonal properties of the characteristic solutions of the system (b) and of its adjoint can be used to obtain the coefficients $c_{k}$ in the representation $F(x)=\Sigma c_{k} Y_{k}(x)$, where the columnar matrices $Y_{k}$ are the characteristic solutions. This representation, with the scalar $c_{k}$ 's computed as stated, is one which well might be expected to be as satisfactory from the viewpoint of convergence as it appears to be from the viewpoint of computation, but it is admittedly formal in general. Whether there are any problems of practical importance that can be treated successfully by Birkhoff and Langer's theory and that, at the same time, do not come within the scope of the paper by Bliss is not known. It does not seem possible to find a proof that the coefficients computed with the use of the adjoint characteristic functions give an expansion that approximates the function in any satisfactory sense in the general case. Use of the characteristic solutions themselves, or of their real parts, gives an expansion which represents the function in the sense of least squares in certain cases but, since the characteristic solutions of the system (b) do not in general form an orthogonal set, the computation of the coefficients requires here the solution of a presumably infinite system of linear equations in the coefficients. The BernoulliFourier problem, that is, the problem of system response to an arbitrary external stress impressed upon a continuous dissipative dynamical system, essentially an expansion problem, thus has had no solution in the general case up to the present. For a nearly arbitrary stress, that is, for a stress for which the Burkhardt transform can be represented by a linear combination of characteristic vector solutions of (b) assumed to have a Green's matrix, a solution is given in the following pages, not only to the Bernoulli-Fourier problem for a system (a) having a Green's matrix but also to the Heaviside problem, that is, the problem of the behavior of a dynamical system subsequent to the sudden imposition of the external forces. The two problems are treated in $\S \S 1$ and 2 , respectively.

Notation. A column matrix, or vector, of elements $u_{1 i}, u_{2 i}, \cdots, u_{n i}$ is designated $u_{i}:$. A line matrix of elements $v_{i 1}, v_{i 2}, \cdots, v_{i n}$ is designated $\cdots v_{i}$. By $\mathscr{W},[w]$, or $\left(w_{i j}\right)$ will be understood a square matrix with element $w_{i j}$ in the $i$ th row and $j$ th column. In vector equations, a column of zeros forming the columnar matrix 0 : is written simply 0 .

The complex number $\mu=\alpha+\iota \omega$ is called a modal number. The $\mu$ 's usually are called characteristic numbers but Hilbert prefers this name for their reciprocals. Since the $\mu$ 's enumerate the modes of motion of a dynamical system, the name modal number seems preferable to "proper number," "Eigenwert" or "characteristic number." 
1. Sustained external forces. A matrix of elements $G_{i j}(x, \xi)$ which are continuous in $x$ except possibly at $x=\xi$ and which have continuous first derivatives in $x$ except at a finite number of points in the square $a \leqq x \leqq b, a \leqq \xi \leqq b$ is called a Green's matrix if it satisfies the steady-state equations for the system (a) and if the elements have the property: $G_{j k}(\xi+0, \xi)-G_{j k}(\xi-0, \xi)=\delta_{j k}$. The existence and uniqueness of the Green's matrix for the system (b) in which $A(x)$ and $B(x)$ are matrices of piecewise continuous elements can be established by a slight and obvious generalization of the proof given by Birkhoff and Langer ${ }^{5}$ where the elements of $A$ and $B$ are assumed to be continuous. A necessary and sufficient condition for the existence of a Green's matrix is that the system (b) with $\mu=0$ have no solution other than $Y \equiv 0$.

The derivative of the Burkhardt transform of an integrable vector requires a more detailed discussion. After both sides of the equation

$$
\int_{a}^{b} G(x, \xi) U(\xi): d \xi=\int_{a}^{x-0} \mathcal{G}(x, \xi) U(\xi): d \xi-\int^{x+0} \mathcal{G}(x, \xi) U(\xi): d \xi
$$

in which $U$ : is any columnar matrix of integrable elements continuous at $x$ have been differentiated, there results

$$
\begin{aligned}
\frac{d}{d x} \int_{a}^{b} G(x, \xi) U(\xi): d \xi= & \int_{a}^{b} \frac{d G(x, \xi)}{d x} U(\xi): d \xi+G(x, x-0) U(x-0): \\
& -G(x, x+0) U(x+0):,
\end{aligned}
$$

an equation in which the last two terms, as a consequence of the continuity of the $G$ 's, may be replaced by

$$
G(x+0, x) U(x):-G(x-0, x) U(x):=\Im U(x):
$$

to give the identity

$$
\frac{d}{d x} \int_{a}^{b} G(x, \xi) U(\xi): d \xi=\int_{a}^{b} \frac{d}{d x} G(x, \xi) U(\xi): d \xi+U(x):
$$

which will be referred to as Lemma I. It may be remarked parenthetically that the more general result

$$
\frac{d^{p+1}}{d x^{p+1}} \int_{a}^{b} G(x, \xi) U(\xi): d \xi=\int_{a}^{b} \frac{d^{p+1}}{d x^{p+1}} G(x, \xi) U(\xi): d \xi+U(x):
$$

\footnotetext{
${ }^{5}$ Ibid., pp. 66-70. Every system (b) has an adjoint in the sense of Bliss but not in the sense of Birkhoff and Langer. In particular, the electrical transmission line grounded at both ends has no adjoint in the latter sense.
} 
holds when

$$
G_{i j}(x, \xi), d G_{i j}(x, \xi) / d x, \cdots, d^{p-1} G_{i j}(x, \xi) / d x^{p-1}
$$

$(i, j=1,2, \cdots, n)$ all are continuous in $x$ and when

$$
\left.\frac{d^{p} G_{i j}(x, \xi)}{d x^{p}}\right|_{\xi-0} ^{\xi+0}=\delta_{i j}
$$

and this generalization has application in those cases (for example, the vibrating string) where fewer equations of individually higher order are taken instead of (a) and for which $U$ : satisfies the equation

$$
U(x, t):=\int_{a}^{b} G(x, \xi)\left\{\mathcal{A}_{1}(\xi) \dot{U}(\xi, t):+\mathcal{A}_{2}(\xi) \ddot{U}(\xi, t):+\cdots\right\} d \xi .
$$

For the existence of modal functions in such cases it is necessary, as can be shown, to assume certain relationships between the $A$ 's with the result that the analysis is not more but less general than that of the equation (1.2) below. In any case, one arrives at the equation (1.8) after the usual Bernoulli-Taylor substitution.

When (a) has a Green's matrix, any solution of the equation

$$
U(x, t):=\int_{a}^{b} G(x, \xi)\left\{\mathcal{A}(\xi) \frac{\partial U(\xi, t):}{\partial t}+E(x, t):\right\} d \xi
$$

is a solution of the nonhomogeneous extension of (a)

$$
\begin{gathered}
\frac{\partial u_{i}}{\partial x}=\Sigma a_{i j}(x) \frac{\partial u_{j}}{\partial t}+\Sigma b_{i j}(x) u_{j}+e_{i}(x, t), \\
\Sigma\left\{\alpha_{i j} u_{j}(a)+\beta_{i j} u_{j}(b)\right\}=0,
\end{gathered}
$$

as may be verified from the properties ${ }^{6}$ of the Green's functions and by virtue of Lemma I. Hence when the arbitrary external force and corresponding response are assumed to have the forms

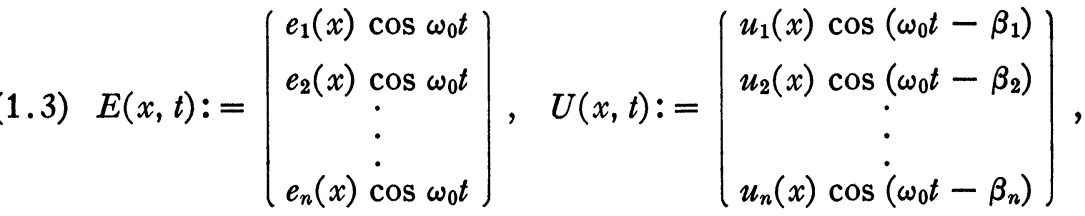

appropriate to a sustained periodic disturbance in a dissipative system governed by (c), substitution into the equivalent equation (1.2) yields the system of integral equations

${ }^{6}$ Bliss, loc. cit., p. 571. 


$$
\left.\begin{array}{l}
W(x):=\omega_{0} \int_{a}^{b} G(x, \xi) A(\xi) X(\xi): d \xi+F(x):, \\
X(x):=-\omega_{0} \int_{a}^{b} G(x, \xi) A(\xi) W(\xi): d \xi
\end{array}\right\}
$$

obtained on letting $t$ take on the values 0 and $\pi / 2 \omega_{0}$, respectively, and where, as matters of notation

$$
\begin{aligned}
W(x):= & \left(\begin{array}{c}
u_{1}(x) \cos \beta_{1} \\
u_{2}(x) \cos \beta_{2} \\
\vdots \\
u_{n}(x) \cos \beta_{n}
\end{array}\right), \quad X(x):=\left(\begin{array}{c}
u_{1}(x) \sin \beta_{1} \\
u_{2}(x) \sin \beta_{2} \\
\vdots \\
u_{n}(x) \sin \beta_{n}
\end{array}\right), \\
& F(x):=\int_{a}^{b} G(x, \xi) E(\xi, 0): d \xi,
\end{aligned}
$$

that is, $F(x):$ is the Burkhardt transform of $E(x, 0)$ :.

The substitution $u_{i}(x, t)=\epsilon^{\alpha t} \cos \left(\omega t-\beta_{i}\right) y_{i}(x)$, appropriate to a free oscillation, into (1.2) with $E: \equiv 0$ gives the homogeneous system

$$
\left.\begin{array}{l}
W(x):=\alpha \int_{a}^{b} K(x, \xi) W(\xi): d \xi+\omega \int_{a}^{b} K(x, \xi) X(\xi): d \xi, \\
X(x):=-\omega \int_{a}^{b} K(x, \xi) W(\xi): d \xi+\alpha \int_{a}^{b} K(x, \xi) X(\xi): d \xi,
\end{array}\right\}
$$

where $K(x, \xi)=G(x, \xi) \mathcal{A}(\xi)$.

The system (1.4) may be written as a single vector equation

$$
U(x):=\mu \int_{a}^{b} K(x, \xi) U(\xi): d \xi+F(x):,
$$

where $U(x):=W(x):-\imath X(x):$ and $\mu=\iota \omega_{0}$ and the system (1.7) may be written in the same way if $\mu=\alpha+\iota \omega, F(x): \equiv 0$.

If $\phi_{r}(x)$ : is a solution of the homogeneous specialization of (1.8), that is, if $\phi_{r}(x)$ : satisfies the equation

$$
\phi_{r}(x):=\mu_{r} \int_{a}^{b} K(x, \xi) \phi_{r}(\xi): d \xi
$$

with $\mu_{r}=\alpha_{r}+\iota \omega_{r}$ for one or more distinct modal numbers $\mu_{r}$ $(r=1,2, \cdots)$ and if $F(x)$ : can be expressed in the form

$$
F(x):=\sum_{r=1}^{l} c_{r} \phi_{r}(x):
$$


where $c_{r}$ is a scalar multiplier, then a particular solution of equation (1.8) is given by the expression

$$
U(x):=\sum_{r=1}^{l} \frac{c_{r} \mu_{r}}{\mu_{r}-\mu} \phi_{r}(x):,
$$

where $l$ may be any natural number no matter how great.

When $n=1$ and the kernel is symmetric, the infinite sums corresponding to (1.10) and (1.11) are known to be convergent in view of results obtained by Schmidt, ${ }^{7}$ and equation (1.11) becomes equivalent to the particular solution of the integral equation of the second kind with symmetric kernel given by him. When the kernel is not symmetric the convergence of the infinite sums in question to the function $F(x)$ : has not been established in any example and, as has been shown by Kowalewski, ${ }^{8}$ the equation (1.9) may have no nonzero solution at all. It can be shown that when the solution $\cdots \psi_{s}$ of the equation following has the property expressed by the equation $\psi_{s}:=\bar{\phi}_{s}$ :, the infinite series $\Sigma c_{r} \phi_{r}(x)$ : converges uniformly and absolutely and represents the Burkhardt ${ }^{9}$ transform $F(x)$ : in the sense of least squares, but this relationship between the $\psi$ 's and $\phi$ 's, although holding in trivial cases, does not hold in general.

If solutions $\cdots \psi_{1}, \cdots \psi_{2}, \cdots, \cdots \psi_{l}$ of the equation

$$
\cdots \psi_{s}(x)=\mu_{s} \int_{a}^{b} \cdots \psi_{s}(\xi) K(\xi, x) d \xi
$$

exist corresponding to the modal numbers $\mu_{1}, \mu_{2}, \cdots, \mu_{l}$ of equation (1.9), then we have the following theorem:

THEOREM I. If the series $\sum_{r=1}^{l} c_{r} \phi_{r}(x):$ is designated by $F(x):$, then

$$
c_{r}=\int_{a}^{b} \cdots \psi_{r}(x) F(x): d x / \int_{a}^{b} \cdots \psi_{r}(x) \phi_{r}(x): d x .
$$

This follows immediately from the easily proved biorthogonality of the sets $\left\{\cdots \psi_{r}\right\},\left\{\phi_{r}:\right\}$. Thus if equation (1.9) with subscript $r$ is premultiplied on both sides by $\cdots \psi_{s}(x)$ and equation (1.12) with subscript $s$ is postmultiplied on both sides by $\phi_{r}(x)$ : there result, after integration,

${ }^{7}$ Erhard Schmidt, Mathematische Annalen, vol. 63 (1907), p. 454

${ }^{8}$ Cf. Love, Integral Equations, p. 117.

${ }^{9} \mathrm{H}$. Burkhardt, Sur les fonctions de Green relatives à un domaine d'une dimension, Bulletin de la Société Mathématique de France, vol. 22 (1894), p. 71. The name "Burkhardt transform" for an expression of the form $\int_{a}^{b} G(x, \xi) e(\xi) d \xi$ is due to H. Bateman. 


$$
\begin{aligned}
& \int_{a}^{b} \cdots \psi_{s}(x) \phi_{r}(x): d x=\mu_{r} \int_{a}^{b} \int_{a}^{b} \cdots \psi_{s}(x) K(x, \xi) \phi_{r}(\xi): d \xi d x \\
& \int_{a}^{b} \cdots \psi_{s}(x) \phi_{r}(x): d x=\mu_{s} \int_{a}^{b} \int_{a}^{b} \cdots \psi_{s}(\xi) K(\xi, x) \phi_{r}(x): d \xi d x
\end{aligned}
$$

two equations in which the double integrals obviously must be zero when $\mu_{s} \neq \mu_{r}$ since they are of equal value.

As a corollary to Theorem I we have this statement: If $F(x)$ : is an arbitrary vector and a set of constants $c_{1}, c_{2}, \cdots, c_{l}$ are computed with the formula of Theorem I, then the series sums to the function or else the function is equal to no linear combination whatever of the l $\phi$ 's.

This corollary says nothing about the possibility of expressing an arbitrary function, or even the Burkhardt transform of an arbitrary function, as a uniformly convergent series in terms of the modal functions, but it is nevertheless of practical importance and is quite adequate to our theoretical needs in those more usual cases where only a limited number of modal functions are known. Suppose $F(x)$ : has been computed from (1.6) for a given force function $E(x, 0)$ : and that a set of constants $c_{1}, c_{2}, \cdots, c_{l}$ has been computed by the formula of Theorem I. Then the function $D(x)$ : given by the equation

$$
D(x):=\Sigma c_{r} \mu_{r} A(x) \phi_{r}(x):
$$

is to be compared with $E(x, 0)$ : and if sufficiently close to $E(x, 0)$ : from a physical standpoint to permit such a restatement of the problem then the function $D(x)$ : is taken as the initially given force function and a particular solution of the equations of motion is given by (1.11) with the $c$ 's as in (1.14). If $D(x)$ : is deemed an inadequate representation of $E(x, 0)$ : and the situation is not improved by enlargement of the sets $\phi_{1}, \phi_{2}, \cdots, \phi_{l}, \psi_{1}, \psi_{2}, \cdots, \psi_{l}$ then there is no solution possible of the kind considered.

2. The Heaviside problem. It was pointed out by Bateman ${ }^{10}$ in 1910 that the Laplace transform of the function $u$ satisfying a partial differential equation of the form

$$
\frac{\partial u}{\partial t}=f\left(\frac{\partial}{\partial x}, \frac{\partial}{\partial y}, \frac{\partial}{\partial z}\right) u
$$

and linear boundary conditions independent of the time will satisfy the same boundary conditions and in general a simpler differential

${ }^{10} \mathrm{H}$. Bateman, Proceedings of the Cambridge Philosophical Society, vol. 15 (1910), p. 423. 
equation provided the value of $u$ at time $t=0$ be known, and application of the theory was made in a heat conduction problem and in a physical problem involving a system of linear differential equations. Since then the Laplace transformation has been resorted to in treatments by many authors ${ }^{11}$ of similar differential equations governing vibration and diffusion problems in which external forces are suddenly imposed at time $t=0$ at one or more regions thereof. In the present section a solution of the problem of the behavior of a dynamical system having a Green's matrix following the sudden application of an external force is obtained as an inversion of a series solution of an integral equation satisfied by the Laplace transform.

When the substitution $u_{i}(x, t)=\epsilon^{\alpha t} \cos \left(\omega t-\beta_{i}\right) y_{i}(x)$ is appropriate to the system (a), $u_{i}(x, t)$ has the Laplace transform $u_{i}^{*}(x, \mu)$ and (c) may be written

$$
\begin{gathered}
\frac{d u_{i}^{*}(x, \mu)}{d x}=\Sigma\left\{\mu a_{i j}(x)+b_{i j}(x)\right\} u_{j}^{*}(x, \mu) \\
+\Sigma a_{i j}(x) u_{j}(x, 0)+e_{i}^{*}(x, \mu), \\
\Sigma\left\{\alpha_{i j} u_{j}^{*}(a, \mu)+\beta_{i j} u_{j}^{*}(b, \mu)\right\}=0,
\end{gathered}
$$

where, as matters of notation,

$$
u_{i}^{*}(x, \mu)=\int_{0}^{\infty} \epsilon^{-\mu t} u_{i}(x, t) d t, \quad e_{i}^{*}(x, \mu)=\int_{0}^{\infty} \epsilon^{-\mu t} e_{i}(x, t) d t .
$$

Assuming, as in Heaviside,

$$
\begin{aligned}
u_{i}(x, t) & =0, & -\infty<t<0, \\
\frac{d e_{i}(x, t)}{d t} & =0, & t>0,
\end{aligned}
$$

equations (2.1) may be written as the vector differential system

$$
\begin{gathered}
\frac{d U_{0}^{*}:}{d x}=\{\mu \mathcal{A}(x)+\mathcal{B}(x)\} U_{0}^{*}+\mu^{-1} E_{0}(x):, \\
{[\alpha] U_{0}^{*}(a, \mu):+[\beta] U_{0}^{*}(b, \mu):=0,}
\end{gathered}
$$

where $E_{0}(x)$ : stands for $E(x, 0+)$ : with matric elements $e_{i}(x, 0+)$ $(i=1,2, \cdots, n)$.

11 The bibliography in Doetsch's book, Theorie und Anwendung der Laplace- Transformation, omits reference to papers and books by van der Pol, Philosophical Magazine, vol. 8 (1929), p. 861; Dalzell, Proceedings of the Physical Society, vol. 42 (1930); Humbert, Le Calcul Symbolique, Paris, 1929; Dahr, Operational Calculus, Stockholm, 1935 , and the paper by Bateman. 
Now $G(x, \xi)$ satisfies, identically in $\xi$, the system

$$
\frac{d G(x, \xi)}{d x}=\mathcal{B}(x) G(x, \xi), \quad[\alpha] G(a, \xi)+[\beta] G(b, \xi)=0
$$

as before and $\int_{a}^{b} G(x, \xi)\left\{\mu \mathcal{A}(\xi) U^{*}(\xi):+\mu^{-1} E_{0}(\xi):\right\} d \xi$ satisfies (2.5) because of the property (1.1); hence equivalent to (2.5) there is the equation

$$
U_{0}^{*}(x):=\mu^{-1} F_{0}(x):+\mu \int_{a}^{b} G(x, \xi) \mathcal{A}(\xi) U_{0}^{*}(\xi): d \xi,
$$

where

$$
F_{0}(x):=\int_{a}^{b} G(x, \xi) E(\xi, 0+): d \xi .
$$

Any solution of (2.7) satisfies (2.5) as may be directly verified. have

When $F_{\tau}(x)$ : can be represented by the sum $\sum_{r=1}^{l} c_{r}(\tau) \phi_{r}(x)$ :, we

$$
U_{0}^{*}(x, \mu):=\sum \frac{\mu_{r} c_{r}(0)}{\left(\mu_{r}-\mu\right) \mu} \phi_{r}(x):
$$

a particular solution of equation (2.7), by the result (1.11), and

$$
U_{0}(x, t):=\frac{1}{2 \pi \iota} \int_{c-\infty \infty}^{c+\infty} \epsilon^{\mu t} U_{0}^{*}(x, \mu): d \mu
$$

by the Riemann-Mellin inversion formula, it being assumed that the $\mu$ 's have negative real part. Dependence of the initial state of the system on the zero of time is indicated by the subscript $\tau$.

When the constant vector $E$ : initially applied at time $t=0$ is abruptly increased at time $t=\tau$ by the increment $\Delta E_{\tau}(x):$, the total response at any time $t>\tau$ is given, in accordance with (2.10), by the equation

$$
U(x, t):=\frac{1}{2 \pi \iota} \int_{c-i \infty}^{c+\infty}\left\{\epsilon^{\mu t} U_{0}^{*}(x, \mu):+\epsilon^{\mu(t-\tau)} \Delta U_{\tau}^{*}(x, \mu):\right\} d \mu
$$

in which

$$
\Delta U_{\tau}^{*}(x, \mu)=\sum \frac{\mu_{r} \Delta c_{r}(\tau)}{\left(\mu_{r}-\mu\right) \mu} \phi_{r}(x): .
$$

It follows, as in the usual applications of Duhamel's theorem, that the response to a variable vector force $E(x, t):$, applied abruptly at 
time $t=0$ but varying in a continuous manner thereafter, is given by the equation

in which

$$
\begin{aligned}
U(x, t):= & \frac{1}{2 \pi \iota} \int_{c-\infty \infty}^{c+\infty}\left\{\epsilon^{\mu t} U_{0}^{*}(x, \mu):\right. \\
& \left.+\int_{0}^{t} \epsilon^{\mu(t-\tau)} \frac{\partial U_{\tau}^{*}(x, \mu):}{\partial \tau} d \tau\right\} d \mu
\end{aligned}
$$

$$
U_{\tau}^{*}:=\sum \frac{\mu_{r} c_{r}(\tau)}{\left(\mu_{r}-\mu\right) \mu} \phi_{r}(x):
$$

as before; $\tau$ is the time of application of the external force. This result is rigorous when $E(x, t)$ : can be expressed in the form $\Sigma c_{r}(t) \mu_{r} \in A(x) \phi_{r}(x)$ : and when $l<\infty$. From physical considerations it seems probable that it is more generally correct.

Of particular interest is the case where $E(x, t)$ : is given by

$$
E(x, t):=e_{0} \sin (\Omega t-\beta) \eta(x): \int_{a}^{b} G(x, \xi) \eta(\xi): d \xi=\sum c_{r} \phi_{r}(x): .
$$

Equation (2.13) here takes the form

$$
U_{r}^{*}(x, \mu):=\sum e_{0} \sin (\Omega t-\beta) \frac{\mu_{r} c_{r}}{\left(\mu_{r}-\mu\right) \mu} \phi_{r}(x):
$$

so that we have

$$
\begin{aligned}
& U(x, t):=\sum \mu_{r} c_{r} \phi_{r}(x): \frac{e_{0} \sin \beta}{2 \pi \iota} \int_{c-\infty \infty}^{c+\infty} \frac{\epsilon^{\mu t}}{\left(\mu-\mu_{r}\right) \mu} d \mu \\
& +\sum \mu_{r} c_{r} \phi_{r}(x): \frac{e_{0} \Omega}{2 \pi \iota} \int_{c-\infty}^{c+\infty} \frac{\epsilon^{\mu t}}{\left(\mu_{r}-\mu\right) \mu} \int_{0}^{t} \epsilon^{-\mu \tau} \cos (\Omega \tau-\beta) d \tau d \mu
\end{aligned}
$$

a solution which reduces, after computation of the integrals, to

$$
\begin{aligned}
U(x, t):= & e_{0} \sum \mu_{r} c_{r} \phi_{r}(x):\left\{\frac{\mu_{r} \sin \beta-\Omega \cos \beta}{\mu_{r}^{2}+\Omega^{2}} \epsilon_{r}^{\mu_{r} t}\right. \\
& \left.+\frac{\mu_{r}}{\mu_{r}^{2}+\Omega^{2}} \sin (\Omega t-\beta)+\frac{\Omega}{\mu_{r}^{2}+\Omega^{2}} \cos (\Omega t-\beta)\right\}
\end{aligned}
$$

a result in which the steady-state and damped oscillations are easily identifiable and in which the characteristic phenomena of resonance are exhibited.

NEW YoRK, N. Y. 